\title{
Anticuerpos anti-albúmina bovina (BSA) en niños diabéticos tipo 1 recién diagnosticados y su asociación con lactancia materna y exposición a leche de vaca
}

\author{
Amaya 0 yarzún $A^{1 a^{*}}, J$ Luis Santos $M^{1 b}$, Elena Carrasco $P^{2 c}$, \\ Cecilia Albala B $^{1}$, Alfonso Salinas $T^{3 a}$, Francisco Pérez $B^{1 b}$. \\ Bovine serum albumin (BSA) \\ antibodies in children with recently \\ diagnosed type 1 diabetes
}

Background: Environmental and genetic factors (viruses, toxins and diet) are involved in the aetiology of type 1 diabetes. Among the dietary factors, the role of breast feeding and exposure to cow's milk proteins deserve special attention. Aim: To determine the anti-BSA-IgG levels in type 1 diabetic children and to analyse the possible association with breast feeding duration, exposure to cow's milk and $\beta$ pancreatic auto-antibodies. Patients and methods: Blood samples were collected from 161 diabetic children and 144 controls to measure anti-BSA-IgG level, GAD65, IA-2 and ICA autoantibodies. All children answered a questionnaire about dietary habits during infancy. Results: anti-BSA-IgG was positive (using a cut off point of $25.6 \mathrm{ng} / \mathrm{ml}$ ) in $98 \%$ of diabetic children and $0 \%$ of the control population. The length of breast feeding or early exposure to cow's milk did not influence the concentration of anti-BSA-IgG. Positive BSA titers did not increase the $\lesssim$ pancreatic reactivity (ICA+, GAD+, IA$2+)$. Conclusions: Our data confirm the high frequency of anti-BSA-IgG among diabetic children. However, a specific role in the immunological process of type 1 diabetes cannot be attributed to this protein (Rev Méd Chile 2003; 131: 865-72).

(Key Words: Breast feeding; Diabetes Mellitus, insulin-dependent; Insulin antibodies; Milk proteins)

\footnotetext{
Recibido el 28 de enero, 2003. Aceptado en versión corregida el 19 de junio, 2003. Trabajo financiado por proyecto FONDECYT \# 1000944.

Programa de Epidemiología Genética, Instituto de Nutrición y Tecnología de los Alimentos (INTA), Universidad de Chile ${ }^{1}$. Unidad de Diabetes, Hospital San Juan de Dios, Facultad de Medicina, Universidad de Chile $^{2}$. Facultad de Química y Biología, Universidad de Santiago de Chile ${ }^{3}$.

aBioquímico

${ }^{b}$ Doctor en Ciencias

cMSc Nutrición

*Tesis de Licenciatura en Bioquímica
}

Correspondencia a: Dr. Francisco Pérez-Bravo. Casilla 138-11.

Santiago- Chile. E mail: fperez@uec.inta.uchile.cl 
T a diabetes mellitus tipo 1 es una enfermedad Lde evolución crónica, de base autoinmune, que se desarrolla en individuos genéticamente susceptibles y que es desencadenada por diversos factores ambientales ${ }^{1}$. En Chile, esta enfermedad ha mostrado un incremento sostenido y progresivo en los últimos 15 años, observándose variaciones en las cifras de incidencia que van desde 2,5/ 100.000 habitantes/año en 1986 a cifras más recientes de 4,5/100.000 habitantes/año en el período 2000. Este incremento es un fenómeno común en todos los países y han hecho que Chile pase de ser considerado de un país de incidencia baja a un país con incidencia intermedia ${ }^{2,3}$.

No se conoce con certeza el mecanismo de destrucción de las células beta-pancreáticas, pero existe amplia evidencia que relaciona la respuesta inmune con una serie de eventos celulares, como la aparición de anticuerpos, entre ellos anticuerpos anti-célula del islote (ICA), anticuerpos anti descarboxilasa del ácido glutámico (GAD65), anticuerpos anti-insulina (IAA), y anticuerpos anti proteína tirosina fosfatasa (IA-2) ${ }^{4-6}$.

En relación a los marcadores de predisposición existe amplia evidencia que da cuenta de la asociación entre determinados alelos del complejo HLA de clase II y la diabetes tipo 1. Investigaciones en diversos grupos étnicos permiten sugerir que la diferencia en la incidencia de la enfermedad podría ser el reflejo de la mayor o menor expresión de moléculas susceptibles o protectoras en cada grupo ${ }^{1,7,8}$. Estudios realizados en Chile, han indicado una menor frecuencia de alelos predisponentes como DR3 o DQB1*0201, en comparación a otras poblaciones con mayor incidencia de diabetes tipo $1^{9-11}$.

El inicio del proceso autoinmune en la diabetes tipo 1 se podría producir mediante un complejo mecanismo inmunológico que involucra ruptura de la tolerancia, mediante la presentación de un péptido diabetogénico 0 a través de mimetismo molecular antigénico ${ }^{4,12-17}$. Cabe destacar que la inducción de la tolerancia involucra un conjunto de eventos y múltiples factores pueden influenciar la capacidad de un antígeno a inducir un estado de tolerancia. Entre éstos se incluyen el sitio de entrada, la dosis, la naturaleza del antígeno, los procesos mucosales coexistentes (permeabilidad, inflamación), el background genético, y la edad del huésped. Altas dosis de antígeno aparecen promocionando la deleción clonal de células $\mathrm{T}$ reactivas como también induciendo apoptosis ${ }^{17}$.

Los primeros estudios experimentales realizados en modelos animales (ratas BB, biobreeding), en los cuales surge la posibilidad de relacionar factores nutricionales con diabetes tipo 1 comenzaron en el año 1984. A partir de ese año, diversos estudios comenzaron a considerar el posible efecto de varias proteínas tales como los presentes en trigo, cebada y leche de vaca sobre la incidencia de esta enfermedad $^{18-20}$. Diversos estudios han revelado la presencia de anticuerpos contra la albúmina bovina (BSA), tanto en ratas BB como en ratones NOD (Non-obese diabetic mice). Estos hallazgos, han podido ser corroborados en el hombre, donde se han encontrado altos títulos de anticuerpos anti-BSA en niños diabéticos de diagnóstico reciente ${ }^{21-27}$.

La lactancia materna corresponde al alimento ideal para favorecer el crecimiento y desarrollo del recién nacido. Durante la lactancia sustituta, el niño que depende de la lactancia artificial dispone de medios muy limitados para hacer frente a los patógenos que ingiera mientras no empiece a producir sus propios complejos de IgA secretoria, los cuales no produce hasta fortalecer su membrana intestinal, la cual resulta ser extremadamente delicada, y un exceso de elementos ajenos podría causar un daño considerable ${ }^{28-30}$.

En este sentido, el propósito de esta investigación ha sido establecer por primera vez en Chile, el nivel de anticuerpos anti-BSA en población diabética de reciente diagnóstico, mediante la estandarización de un sistema de ELISA. Comparar dichos títulos con población control no diabética y establecer posibles asociaciones entre el perfil de anticuerpos anti-BSA y factores ambientales, tales como la duración de la lactancia materna exclusiva y la exposición a leche de vaca, con el objetivo de intentar comprender el papel que tendría este anticuerpo «dietario»en la etiopatogenia de la enfermedad.

\section{Pacientes y MÉtodos}

Identificación y selección de los individuos. El estudio corresponde a un diseño caso-control que fue llevado a cabo en Santiago de Chile durante el período 1999-2002, recolectando muestras de ca- 
sos de diabetes tipo 1 recién diagnosticados (hasta después de 6 semanas del debut), menores de 15 años, y dependientes absolutos de insulina exógena, obtenidos de diversas unidades asistenciales en hospitales de Santiago. El grupo de niños controles se seleccionó de 3 colegios de Santiago y consideró niños de ambos sexos cuyas características demográficas (comunas), situación socioeconómica, edad y sexo fueran comparables a los niños provenientes del registro de casos nuevos de diabetes tipo 1. En el grupo control, se seleccionaron aquellos participantes que no presentaban antecedentes familiares de diabetes tipo 1 , ni antecedentes de patologías autoinmunes en el núcleo familiar.

Se recopiló información en 161 casos con diabetes tipo $1(8,2 \pm 4,0$ años) y en un grupo de 144 niños controles $(9,2 \pm 3,0$ años). Los padres de los niños contestaron una encuesta familiar que incluyó información con respecto a historia familiar de diabetes, lactancia materna exclusiva, enfermedades virales, primer consumo de leche de vaca, sustituto lácteo y sólidos lácteos. Todos los participantes entregaron un consentimiento escrito, siendo ésta una investigación aprobada por el comité de ética del INTA.

Con el fin de establecer una posible asociación entre anticuerpos anti-BSA-IgG y la lactancia materna exclusiva o la exposición a la leche de vaca, se analizaron dos variables, a) los meses de duración de la lactancia materna (en categorías $\leq 3$ meses y >3 meses), lo que nos indica cuánto tiempo fue amamantado un niño de forma exclusiva y b) el mes de introducción de la fórmula láctea que tiene relación con el mes que dejó de ser exclusiva la lactancia materna y el niño tuvo su primer encuentro con proteínas foráneas, específicamente leche de vaca. Se establecieron categorías para la medición de anti-BSA-IgG y para ello se utilizó como valor mínimo el de dos desviaciones estándar $(25,6 \mathrm{ng} / \mathrm{ml})$ observado en la distribución de los casos. Datos menores a este valor fueron catalogados $\mathrm{BSA}^{-}$y superiores como $\mathrm{BSA}^{+}$.

Cuantificación de autoanticuerpos anti-GAD, ICA e IA-2. Se determinaron los anticuerpos anticélula del islote (ICA), por inmunofluorescencia indirecta mediante un kit comercial de la compañía IMMCO-Diagnostics (USA). Se consideraron ICA $^{+}$ a aquellas muestras que presentaban valores mayores o iguales a 20 unidades JDF. Los anticuerpos anti-GAD65 y anti-IA-2 se midieron por radioinmunoanálisis (RIA), mediante kit comercial de la empresa Cis-International (Francia). Se consideraron $\mathrm{GAD}^{+}$o IA-2 ${ }^{+}$a aquellos valores superiores a $1 \mathrm{U} / \mathrm{ml}$. Los coeficientes de variación intraensayo e interensayo fueron de $2,1 \%$ y $3,5 \%$, respectivamente.

Cuantificación de anticuerpos anti-BSA. A partir de la muestra de suero obtenida por centrifugación se realizó el siguiente protocolo. Se agregó a cada pocillo $200 \mu \mathrm{L}$ de BSA Sigma ${ }^{\circledR}$, en medio PBS $0,1 \mathrm{~mol} / \mathrm{L} \mathrm{pH} \mathrm{7,4.} \mathrm{Se} \mathrm{incubó} \mathrm{toda} \mathrm{la}$ noche a $25^{\circ} \mathrm{C}$. Al día siguiente, se lavaron las placas con PBS para luego adicionar $370 \mu \mathrm{L}$ de gelatina-PBS al $1 \%$ llevando a incubación a $37^{\circ} \mathrm{C}$ durante un período de 1 hora. Cada muestra se diluyó 1:40 los controles, y 1:80 para los casos en gelatina-PBS-Tween $20^{\circledR}(0,5 \%-100 \%-0,05 \%)$. Se agregaron $200 \mu \mathrm{L}$ de cada muestra por duplicado, blanco y curva de calibración o calibrador. Se dejó incubar por $60 \mathrm{~min}$ a $37^{\circ} \mathrm{C}$ y luego se lavaron las placas varias veces con Tween $20^{\circledR} 0,05 \%$. Posteriormente, se agregó $50 \mu \mathrm{L}$ del conjugado anti IgG 1:2000 marcado con peroxidasa de rábano (Sigma, A6029, St Luis, MO). Finalmente se realizó una incubación por $60 \mathrm{~min}$ a $25^{\circ} \mathrm{C}$ y un último lavado con PBS. Se agregaron $50 \mu \mathrm{L}$ de OPD $0,4 \mathrm{mg} / \mathrm{ml}$, $0,001 \% \mathrm{v} / \mathrm{v}$ de $\mathrm{H}_{2} \mathrm{O}_{2}$ a los $10 \mathrm{~min}$. La reacción se detuvo por adición de $100 \mu \mathrm{L}$ de $\mathrm{HCl} 3 \mathrm{~N}$ por pocillo. Luego se leyó a $492 \mathrm{~nm}$ en un lector de placas. El coeficiente de variación intraensayo e interensayo fue de 1,6 y 4,3\%, respectivamente, el límite de detección fue de $2,6 \mathrm{ng} / \mathrm{ml}$ con una sensibilidad de $0,02 \mathrm{ng} / \mathrm{ml}$.

Estadística. Se utilizaron las pruebas estadísticas U de Mann Whitney y Kruskal-Wallis para comparar la duración de la lactancia e incorporación de leche de vaca, con anticuerpos anti-BSA IgG en $\mathrm{ng} / \mathrm{ml}$. La prueba de Chi cuadrado fue utilizada para establecer las diferencias de asociación entre las distintas categorías.

\section{RESULTADOS}

a) Distribución de anticuerpos anti-BSA-IgG. Para analizar la distribución de los anticuerpos anti- 
BSA IgG, se construyó un gráfico que muestra a los controles $(n=144)$ y a los casos $(n=161)$, agrupados por categorías, según sus niveles de anti-BSA-IgG (Figura 1). En la Figura 1a se puede observar que la distribución del anti-BSA-IgG de los casos posee un comportamiento normal o gaussiano. En la Figura 1b observamos una distribución completamente diferente en los controles.

La dispersión de los valores tanto en los casos, como en los controles se puede apreciar en la Tabla 1 a partir de la cual se puede observar el amplio rango de distribución y una diferencia significativa ( $p<0,0001$ ) al comparar el título promedio entre niños diabéticos y niños controles. b) Asociación entre anticuerpos anti-BSA y exposición a la leche de vaca. La Tabla 2 muestra cómo se distribuye el anticuerpo anti-BSA-IgG de acuerdo al ingreso de la fórmula láctea ( $>3$ meses y $\leq 3$ meses) observándose en los casos, que este título no se modifica si se considera el ingreso a la fórmula láctea en cualquiera de estos dos puntos de corte. Por el contrario, en el grupo control, existe una diferencia significativa $(p<0,003)$ encontrándose un mayor título en aquellos niños que recibieron lactancia sustituta después de los 3 meses.

La Tabla 3, indica que al realizar un cálculo del tiempo de exposición a la fórmula láctea (meses

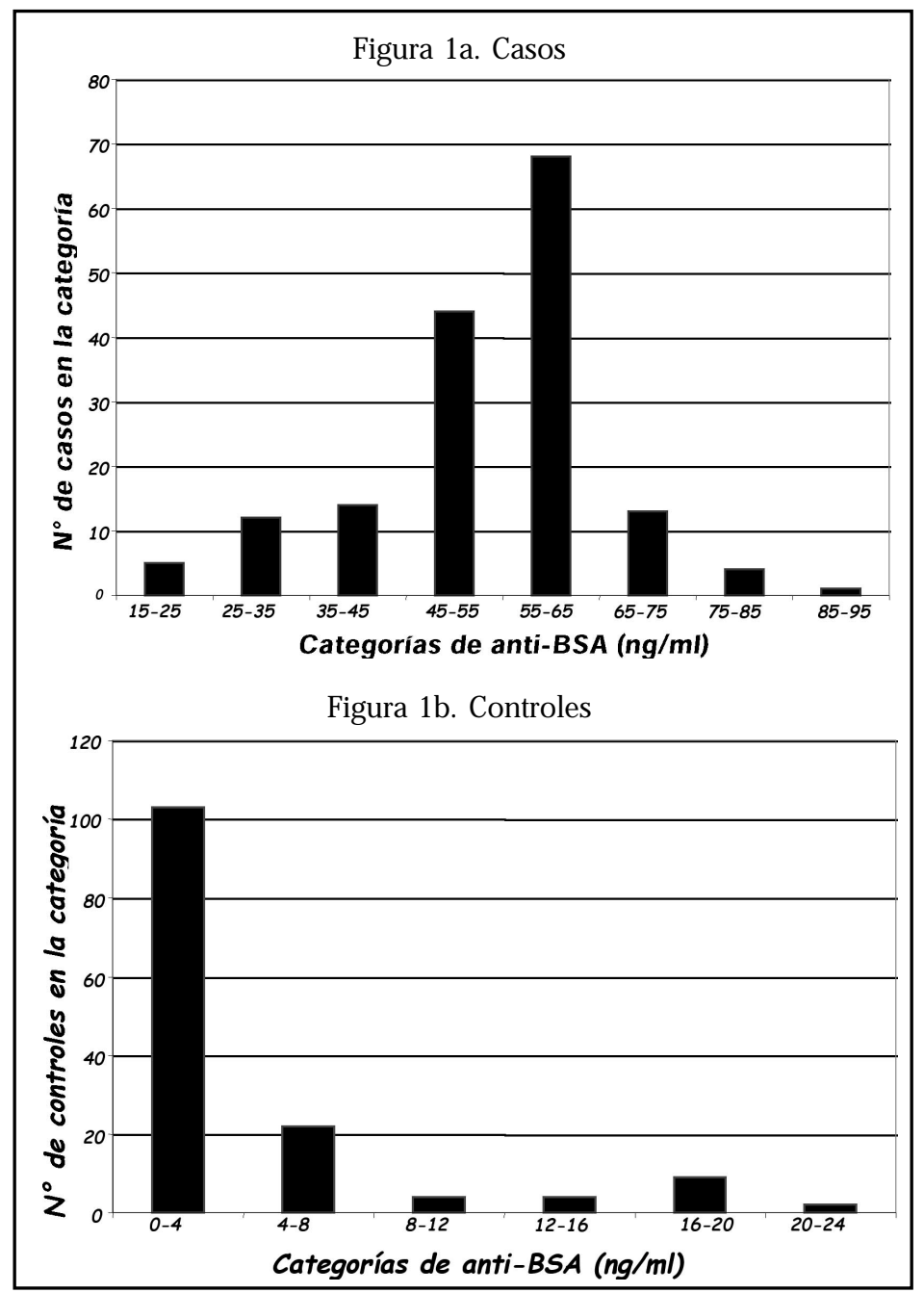

Figura 1. Distribución del anticuerpo anti-BSA-IgG en niños diabéticos y controles ordenados según rangos de concentración del anticuerpo. 
Tabla 1. D istribución de anticuerpos anti-BSA-IgG en niños diabéticos de diagnóstico reciente y niños controles

\begin{tabular}{|lcc|}
\hline & $\begin{array}{c}\text { Anti-BSA-IgG } \\
(\mathrm{ng} / \mathrm{ml})\end{array}$ & Rango \\
\hline Diabéticos $(\mathrm{n}=161)$ & $53,1 \pm 12,8$ & $15,3-94,2$ \\
Controles $(\mathrm{n}=144)$ & $7,8 \pm 6,0^{*}$ & $2,6-21,4$ \\
\hline
\end{tabular}

$* p<0,0001$.

Tabla 2. Concentración del anticuerpo anti-BSA-IgG en relación al período de inicio de lactancia con fórmula láctea

\begin{tabular}{|ccccc|}
\hline & \multicolumn{2}{c}{ Diabéticos } & \multicolumn{2}{c|}{ Controles } \\
& $\mathrm{n}$ & $\mathrm{ng} / \mathrm{ml}$ & $\mathrm{n}$ & $\mathrm{ng} / \mathrm{ml}$ \\
\hline$\leq 3$ meses & 62 & $54,4 \pm 12,5$ & 64 & $1,5 \pm 3,9$ \\
$>3$ meses & 89 & $52,8 \pm 13,4$ & 41 & $5,0 \pm 7,9^{*}$ \\
\hline
\end{tabular}

$*_{p}=0,003$

Tabla 3. Mes de exposición a la fórmula láctea en diabéticos y controles según categorización del anticuerpo anti-BSA (valores en mediana y rango)

\begin{tabular}{|lcccc|}
\hline & \multicolumn{2}{c}{ Diabéticos } & \multicolumn{2}{c|}{ Controles } \\
& $\mathrm{n}$ & meses & $\mathrm{n}$ & meses \\
\hline BSA+ & 145 & $4(0-24)$ & 0 & - \\
BSA- & 6 & $4(0-24)$ & 108 & $3(0-8)$ \\
\hline
\end{tabular}

expresados en mediana y rango) de acuerdo a la categoría BSA+ o BSA-, no se observaron diferencias en las medianas para el grupo de diabéticos, indicando que el mes de exposición no influiría en la categorización del anti-BSA. En el grupo control y dado el punto de corte utilizado en la definición para el perfil anti-BSA-IgG, no se encontraron niños en la categoría BSA+.
Tabla 4. Concentración del anticuerpo anti-BSA-IgG en relación al período de lactancia materna exclusiva

\begin{tabular}{|ccccc|}
\hline & \multicolumn{2}{c}{ Diabéticos } & \multicolumn{2}{c|}{ Controles } \\
& $\mathrm{n}$ & $\mathrm{ng} / \mathrm{ml}$ & $\mathrm{n}$ & $\mathrm{ng} / \mathrm{ml}$ \\
\hline$\leq 3$ meses & 43 & $54,1 \pm 13,7$ & 32 & $0,5 \pm 2,2$ \\
$>3$ meses & 108 & $53,2 \pm 12,8$ & 73 & $4,3 \pm 7,3^{*}$ \\
\hline
\end{tabular}

${ }^{*} \mathrm{p}=0,004$

Tabla 5. Categorías del anti-BSA-IgG y duración de la lactancia exclusiva (mediana y rango) en niños diabéticos y controles

\begin{tabular}{|lcccc|}
\hline & \multicolumn{2}{c}{ Diabéticos } & \multicolumn{2}{c|}{ Controles } \\
& $\mathrm{n}$ & meses & $\mathrm{n}$ & meses \\
\hline BSA+ & 145 & $6(0-24)$ & 0 & - \\
BSA- & 6 & $6(0-24)$ & 106 & $5(0-12)$ \\
\hline
\end{tabular}

c) Asociación entre anticuerpos anti-BSA y lactancia materna exclusiva. En las Tablas 4 y 5 se analiza la variable lactancia materna exclusiva bajo las mismas condiciones anteriores, de esta forma se observa que la duración de la lactancia materna no modifica los títulos del anticuerpo anti-BSA-IgG en el grupo diabético y que sorprendentemente aquellos niños controles que recibieron menor lactancia materna exclusiva poseen un título más bajo de este anticuerpo antiBSA al compararlos con aquellos controles que recibieron lactancia materna exclusiva prolongada ( $p<0,004)$. El análisis observado en la Tabla 5 indica que el mes de duración de la lactancia materna exclusiva no fue diferente según la categoría del anticuerpo $\left(\mathrm{BSA}^{+} \mathrm{o} \mathrm{BSA}^{-}\right)$en el grupo de diabéticos. Esta observación no se pudo realizar en el grupo control, dado que no existe la categoría $\mathrm{BSA}^{+}$.

d) Anticuerpos anti-BSA y perfil de anticuerpos pancreáticos. Para determinar una posible asociación entre los niveles de anti-BSA-IgG y el perfil de anticuerpos pancreáticos, se calcularon los 
porcentajes de $\mathrm{ICA}^{+}, \mathrm{GAD}^{+}$, e $\mathrm{IA}-2^{+}$en el grupo total de diabéticos y luego estratificados por la categoría $\mathrm{BSA}^{+}$.

En la Tabla 6 se puede observar, que el porcentaje de positividad del grupo total casi no se modifica al compararlos con la categoría BSA ${ }^{+}$.

Tabla 6. Porcentaje de positividad de los anticuerpos ß pancreáticos de acuerdo a cada categoría de anti-BSA-IgG en niños diabéticos

\begin{tabular}{|lcc|}
\hline Autoanticuerpo & $\begin{array}{c}\text { BSA+y BSA- } \\
(\mathrm{n}=153)\end{array}$ & $\begin{array}{c}\text { BSA+ } \\
(\mathrm{n}=127)\end{array}$ \\
\hline ICA+ & $84,3 \%$ & $83,1 \%$ \\
GAD+ & $65,4 \%$ & $55,9 \%$ \\
IA- + & $60,4 \%$ & $60,6 \%$ \\
\hline
\end{tabular}

ICA+ = toda medición superior a $20 \mathrm{JDF}$

$\mathrm{GAD}+\mathrm{e} \mathrm{IA}-2+=$ toda medición superior a $1 \mathrm{U} / \mathrm{ml}$

\section{DiscUSIÓN}

La respuesta inmune frente a elementos dietarios, ha sido uno de los campos más controversiales en el análisis de los factores ambientales que podrían gatillar la diabetes tipo $1^{5,17}$. Uno de los puntos más importantes en el estudio de la patogénesis de la enfermedad, es el entender por qué en un momento determinado se desarrolla el proceso autoinmune, cuáles son las células y moléculas que participan, el orden de aparición de éstas, cuáles son las señales que se interrelacionan y finalmente, qué elementos determinarán cuáles son los mecanismos intracelulares que dañan específicamente a la célula $§$ pancreática.

Los resultados anteriormente descritos nos muestran un altísimo título de anti-BSA-IgG en pacientes diabéticos de reciente diagnóstico; fenómeno descrito en múltiples estudios ${ }^{18-29}$, pero que hasta la fecha no tiene una explicación convincente. El alto título de anticuerpos anti-BSA detectado en nuestra población diabética, demuestra que hubo un traspaso del péptido o parte de él, a través de la membrana mucosa intestinal y una posterior respuesta inmunitaria. Especulando sobre este tema, se podrían plantear dos posibles interpretaciones: la primera que indique que este evento ocurrió en una edad muy temprana en que la mucosa intestinal no se encuentra completamente madura y recubierta, y el BSA o un segmento de éste (péptido ABBOS) traspasó esta barrera generando una respuesta inmune, la cual por mimetismo molecular destruyó la proteína de membrana ICA69, con su consecuente daño $\beta$ pancreático ${ }^{18,21}$. Si esto fuera así, se esperaría un alto nivel de anti-BSA-IgG en pacientes que fueron poco amamantados, o que recibieron fórmulas lácteas a muy temprana edad, sin embargo nuestro estudio no observó variaciones con respecto a la asociación de este título de anti-BSAIgG y estas dos variables. De este modo, una explicación alternativa frente al proceso que podría estar ocurriendo sería que en cualquier momento de la vida en que se consume leche de vaca, las mismas características del paciente diabético (susceptibilidad genética, carga viral y otros gatillantes medioambientales), propenden a que el BSA o un segmento de éste traspase la mucosa intestinal y no exista tolerancia hacia este péptido. Puede ser que si en este período ya existen anticuerpos anti-ICA69 éstos se unan a BSA de forma incompleta, y sin destruir a la proteína, cambien su estructura o dejen expuestas otras cadenas peptídicas generando anticuerpos. De este modo, se exacerbaría la respuesta inmune acortando los tiempos de destrucción de las células $ß$ del páncreas. Según esta explicación no veríamos cambios con respecto a la lactancia materna o al ingreso de la fórmula láctea; tampoco nos encontraríamos con un nivel de autoanticuerpos más elevados que de costumbre, y explicaría la asociación que se observa entre los niños con altos niveles de anti-BSA-IgG y genotipos de alto riesgo 28,30 .

Nuestros datos confirman la alta frecuencia de anti-BSA-IgG en la población diabética; sin embargo, no es posible atribuir a esta proteína un papel específico en el desarrollo de autoinmunidad $\beta$ pancreática, siendo posiblemente un evento inmunológico posterior a los estadios iniciales de la diabetes tipo 1. 


\section{REFERENCIAS}

1. FIELD L. Genetic linkage and association studies of Type I diabetes: challenges and rewards. Diabetología 2002; 45: 21-35.

2. Carrasco E, Pérez-Bravo F, Santos JL, López G, Calvilán M, WolfF C, García de los Ríos M. One of the lowest validated incidence rates of insulin dependent diabetes mellitus in the Americas: Santiago, Chile. Diab Res Clin Pract 1996; 34 (Suppl): 153-7.

3. Carrasco E, Pérez-Bravo F, Dorman J, Mondragón A, SANTOS JL. Incidence rate of type 1 diabetes in Santiago (Chile): a 15 years study. Rev Cubana Endocrinol 2002; 13: 72-3.

4. XiAO B, LiNK H. Mucosal Tolerance: A Two-Edged Sword to Prevent and Treat Autoimmune Diseases. Clin Immunol Immunopathol 1997; 85: 119-28.

5. Rosenbloom A, Schatz D, Krischer J, Skyler J, Becker D, Laporte R, Libman I et al. Prevention and Treatment of Diabetes in Children. JCEM 2000; 85: 494-506.

6. Hummel M, Füchtenbusch M, Schenker M, Ziegler A. No Major Association of Breast Feeding, Vaccinations, and Childhood Viral Diseases with Early Islet Autoimmunity in the German BABYDIAB Study. Diabetes Care 2000; 23: 969-74.

7. Park Y, Eisenbarth G. Genetic susceptibility factors to Type 1 diabetes in Asians. Diabetes Metab Res Rev 2001; 17: 2-11.

8. Unduen D, Lie B, ThorsBy E. HLA complex genes in type 1 diabetes and other autoimmune diseases. Which genes are involved? TRENDS Genet 2001; 17: 93-100.

9. Pérez-Bravo F, Serrano-Ríos $\mathrm{M}$, Gutiérrez-López MD, Calviláa M, García de los Ríos M, Kocova M ET AL. Genetic analysis of HLA DRB1, DQA1 and DQB1 alleles and susceptibility to Type I (insulin dependent) diabetes mellitus in Chilean subjects. Diabetologia 1995; 38: 378-9.

10. Santos J, Pérez-Bravo F, Carrasco E, Calvilán M, Albala C. Association between HLA-DQB1 Alleles and Type 1 Diabetes in a Case Parents Study Conducted in Santiago, Chile. Am J Epidemiol 2001; 153: 794-8.

11. Santos J, Pérez-Bravo F, Carrasco E, Petri R, Calvilán M, Albala C. Associations between HLADQB1 high risk alleles and Type 1 Diabetes do not depend on cytomegalovirus antibody status at onset: A case parent study conducted in Chile. Immunol Cell Biol 2000; 78: 259-63.

12. Pozziш P. ß-casein in cow's milk; a mayor antigenic determinant for type 1 diabetes? J Endocrinol Invest 1999; 22: 562-7.

13. Paronen J, Knip M, Savilahti E, Virtanen S, Ilonen J, AKerBLom HK et aL. Effect of Cow's Milk Exposure and Maternal Type 1 Diabetes on Cellular and Humoral Immunization to Dietary Insulin in Infants at Genetic Risk for Type 1 Diabetes. Diabetes 2000; 49: 1657-65.

14. Dosch H, Cheung R, Karges W, Pietropaolo M, BeCKer D. Persistent T Cell Anergy in Human Type I Diabetes. J Immunol 1999; 163: 6933-40.

15. Mayer A, Rharbaoui, Thivolet C, Orgiazzi J, Madec A. The Relationship between Peripheral T Cell Reactivity to Insulin, Clinical Remissions and Cytokine Production in Type 1 (Insulin-Dependient) Diabetes Mellitus. JCEM 1999; 84: 2419-24.

16. Karlsson M, García J, Ludvigsson J. Cows' milk proteins cause similar Th1 and Th2 like immune response in diabetic and healthy children. Diabetología 2001; 44: 1140-7.

17. MaYeR L. Oral Tolerance: New Approaches, New Problems. Clin Immunol 2000; 94: 1-8.

18. Kunreja A, Maclaren N. Current cases in which epitope mimicry is consedered as a component cause of autoimmune disease: immune mediated (type 1) diabetes. Cell Mol Life Sci 2000; 57: 53441.

19. Harrison L, Honeyman M. Cow's Milk and Type I Diabetes. Diabetes 1999; 48: 1501-7.

20. Monetini L, Cavallo M, Stefanini L, Ferrazzol F, BizzaRRi C, Marietti G ET aL. Bovine ß-casein antibodies in breast and bottle feeding infants: their relevance in Type 1 diabetes. Diabetes Metab Res Rev 2001; 17: 51-4.

21. Schrezenmeir J, Jagia A. Milk and Diabetes. J Am Coll Nut 2000; 19: 176S-190S.

22. Paronen J, Björksten, Hattevig G, Åkerblom $H$, VAarala O. Effect of maternal diet during lactation on development of bovine insulin binding antibodies in children at risk for allergy. J Allergy Clin Immunol 2000; 106: 302-6.

23. KimpimäKi T, ERKKola M, Korhonen S, Kupila A, VirTanen S, ILonen J et aL. Short term exclusive breast feeding predisposes young children with increased genetic risk of Type I diabetes to progressive beta cell autoimmunity. Diabetología 2001; 44: 63-9. 
24. Norris J, Pietropaolo M. Controversial topics series: Milk proteins and diabetes. J Endocrinol Invest 1999; 22: 568-80.

25. Couper J, Steele C, Beresford S, Powell T, McCaul K, Polnard A ET AL. Lack of Association Between Duration of Breast Feeding or Introduction of Cow's Milk and Development of Islet Autoimmunity. Diabetes 1999; 48: 2145-9.

26. Karlsson M, LudvigSSON J. The ABBOS peptide from bovine serum albumin causes an IFNgamma and IL-4 mRNA response in lymphocytes from children with recent onset of type I diabetes. Diab Res Clin Pract 2000; 47: 199207.

27. Hilger C, Grigioni F, Beaufort C, Michel G, FreiLnger J, Hentges F. Differential binding of IgG and IgA antibodies to antigenic determinants of bovine serum albumin. Clin Exp Immunol 2001; 123: 387-94.

28. Sarugeri E, Dozio N, Meschi F, Rocco M, Bonifacio E. Cellular and Humoral Immunity against Cow's Milk Proteins in Type 1 Diabetes. J Autoimmunity 1999; 13: 365-73.

29. Virtanen S, LäÄrä E, Hyppönen E, Reijonen H, RäsÄNEN L, ARO A ET AL. Cow's Milk Consumption, HLA-DQB1 Genotype, and Type 1 Diabetes. Diabetes 2000; 49: 912-7.

30. Vaarala O, Knip M, Paronen J, Muona P, Ilonen J, Simell O et al. Cow's Milk Formula Feeding Induces Primary Immunization to Insulin in Infants at Genetic risk for Type 1 Diabetes. Diabetes 1999; 48: 1389-94.

\section{Agradecimientos}

Los autores agradecen la colaboración de todas las familias participantes de esta investigación. Asimismo a los Hospitales San Juan de Dios, San Borja Arriarán, Exequiel González Cortés, Roberto del Río y Clínica Alemana. Además, agradecemos la buena disposición de la Fundación de Diabetes Juvenil de Chile. 